\title{
MATRIZ PRODUCTIVA Y EL MODELO DE SUSTITUCIÓN DE IMPORTACIONES DEL SECTOR TEXTIL ECUATORIANO (2009- 2017) CASO DE ESTUDIO: CANTÓN ANTONIO ANTE
}

\author{
Marcela Fernanda Coronel Pinduisaca ${ }^{1 *}$, Andrea Celina Echeverría Cueva $^{2}$ \\ ${ }^{1}$ Pontificia Universidad Católica del Ecuador Sede Ibarra Escuela de Negocios y Comercio Internacional mfcoronel@pucesi.edu.ec \\ ${ }^{2}$ Ingeniera en Comercio Internacional, acecheverria@pucesi.edu.ec
}

* Autor para correspondencia: mfcoronelucesi.edu.ec, 0997901905

Recibido: 2019/09/23

Aprobado: 2020/11/07

DOI: https://doi.org/10.26621/XVI23.2020.12.A06.PUCESI.2550.6684

\section{RESUMEN}

La CEPAL a través de sus postulados establece ideales que logren fomentar el cambio de la matriz productiva, permitiendo el crecimiento económico de los países en desarrollo, es así que Ecuador a través de instrumentos políticos y comerciales da inicio a la transformación del sector productivo, donde el Plan Nacional, la Agenda para la Transformación Productiva, la Política Industrial y Estrategias, establecen lineamientos que contribuyan a sectores priorizados con incentivos de índole; comercial, financiera, promocional, productiva y tributaria, que incentiven la industria textil y promuevan un adecuado cambio de la matriz productiva. La industria textil nacional posee importancia en el sector económico por ser generadora de empleo, es la segunda industria manufacturera que proporciona innovación y cuenta con alto valor agregado. A nivel de la provincia de Imbabura se ha seleccionado como caso de estudio al cantón Antonio Ante, al representar una historia cultural textil, con el objetivo de establecer información sobre la estructura y comportamiento que posee el sector, con el involucramiento de los actores responsables del cumplimiento, de lo establecido en el Plan Nacional del Buen Vivir del 2009 al 2013 y 2013 al 2017. La investigación aborda la aplicación de las políticas Estatales en pro del beneficio del sector productivo textil y cómo los actores directos han sabido aprovechar estas oportunidades, o si por el contrario este sector sigue siendo el más vulnerable.

Palabras clave: textil, matriz productiva, políticas estatales, Antonio Ante

\section{ABSTRACT}

ECLAC, through its postulates, establishes ideals that manage to promote the change of the productive matrix, allowing the economic growth of developing countries, this is how Ecuador, through political and commercial instruments, began the transformation of the productive sector, where the National Plan, the Agenda for Productive Transformation, the Industrial Policy and Strategies, establish guidelines that contribute to prioritized sectors with incentives of a commercial, financial, promotional, productive and tax nature, that It encourages the textile industry and promote an adequate change in the productive matrix. The national textile industry is important in the economic sector because it generates employment, It is the second manufacturing industry that It provides innovation and has high added value. At the level of Imbabura Province, Antonio Ante canton has been selected as a case study, as it represents a cultural textile history, with the objective of establishing information on the structure and behavior that the sector has, with the involvement of the actors responsible for compliance, of what is established in the National Plan for Good Living from 2009 to 2013 and 2013 to 2017. The research addresses the application of policies states for the benefit of the productive textile sector and how the direct actors have known how to take advantage of these opportunities, or It is the sector that It continues to be the most vulnerable.

Keywords: textile, productive matrix, state policies, Antonio Ante

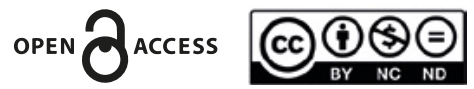




\section{INTRODUCCIÓN}

Su busca demostrar el impacto de la transformación de la matriz productiva en el sector textil, esencialmente en la industria manufacturera del cantón Antonio Ante, con la ejecución de las políticas públicas del Estado ecuatoriano por los actores de este sector. Si bien esta política de Estado tiene algunos años de implementación, resulta menester indagar ¿cuál ha sido el efecto que ha tenido el cambio de la matriz productiva en los distintos sectores económicos del país? En este contexto y para adecuar la pregunta de investigación a un objeto de estudio, se ha tomado específicamente al sector textil. Se tiene en cuenta que la provincia de Imbabura es la quinta región manufacturera con el $2.11 \%$ de participación a nivel nacional (INEC, 2012) y que, según datos del Banco Central del Ecuador, la manufactura es la tercera actividad económica después de la agricultura, ganadería, silvicultura y pesca y la explotación de minas y canteras. (Garzón , Kulfas, Palacios, \& Dirchelmo, 2016)

Para el desarrollo de esta investigación se ha analizado el postulado de Prebisch y la Teoría de la Dependencia que tienen un objetivo común de lograr; desarrollo, crecimiento y competitividad en los factores socioeconómicos de los países en desarrollo, especialmente de América Latina y el Caribe, (Prebisch, 2012) a través de las estrategias de trasformación o cambio de la matriz productiva, en los sectores considerados estratégicos, como es el caso del sector textil, el cual representa una industria que participa en el contexto mundial.

El sector textil destina parte de la producción al consumo nacional, lo que motiva la búsqueda de acuerdos comerciales que satisfagan las dos partes, al promover el crecimiento y desarrollo de la industria, se permite la búsqueda de estrategias para que las empresas busquen establecerse en el mercado internacional, cubriendo la demanda insatisfecha. La industria textil del cantón Antonio Ante considera que la firma de acuerdos comerciales con Estados Unidos, benefician al sector de manera precisa al ser el mayor proveedor de materia prima e insumos, para el proceso de fabricación, permitiría disminuir costos de compra, logrando aprovechar los objetivos y estrategias establecidas para el adecuado cambio de la matriz productiva, a través de productos de calidad y valor agregado, satisfaciendo la necesidad del consumidor externo. La experticia del sector textil en el cantón Antonio Ante, permite cubrir la necesidad del proceso productivo, pues cumple la función de participar en su mejora a través de la innovación. Sin embargo, ha surgido un conflicto de competencia desleal por parte de los pequeños productores, la falta de ayuda de organismos gubernamentales provoca una falta de aprovechamiento de los instrumentos que establece el gobierno.

Han existido investigaciones relacionadas con el sector textil ecuatoriano y sobre cómo se ha dado su incidencia en el área de la manufactura, Portilla Pinto en el año 2015 realiza un análisis de los rendimientos crecientes en este sector, como resultado Pinto establece que una de las debilidades es el tener poco personal calificado, lo que incrementa la desventaja competitiva por falta de valor agregado e innovación, cuando de por medio se fija como meta exclusiva la reducción de costos.

\section{MATERIALES Y MÉTODOS}

El enfoque del estudio que se propone es de tipo mixto (tanto cualitativo como cuantitativo), se describe y analiza postulados teóricos y técnicos de carácter cualitativo que busca analizar la problemática de la investigación, a través de un estudio de campo dirigido a la Feria Textil realizada del 1 al 5 de marzo del 2019. En relación al enfoque cuantitativo y cualitativo la aplicación de entrevistas a los responsables del departa- mento de Gestión de Desarrollo del GAD Municipal de Antonio Ante, permitió contar con la base catastral y posterior delimitación de la muestra.

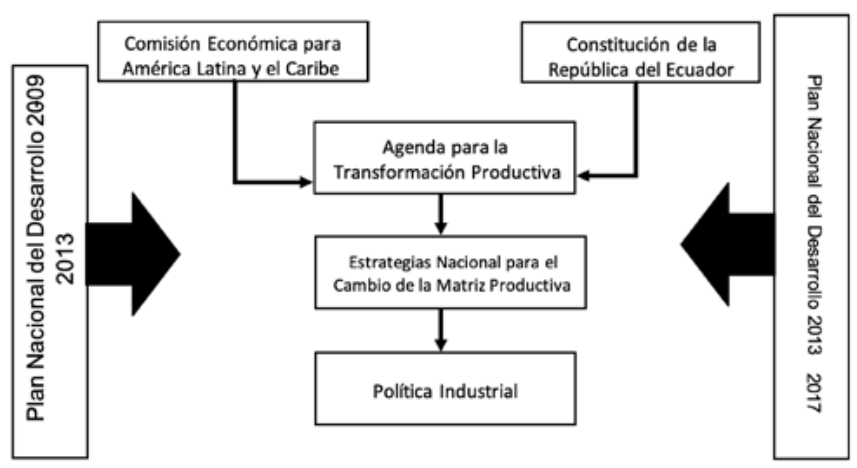

Figura 1. Participación de actores

\section{Métodos}

La recolección de información es cuanti-cualitativo y se desarrolla a través de entrevistas, a los diversos actores de las actividades económicas que se desempeñan en el catón, a través de la utilización del método estratificado donde se encontrará la representatividad de la industria textil con la división de subgrupos según la figura legal que presentan las empresas, es decir naturales o jurídicas.

\section{Población y muestra}

Los datos proporcionados por el Departamento de Rentas Internas del GAD Municipal del cantón Antonio Ante brindan información accesible para el cálculo de muestra. A continuación, se indica el cálculo de la muestra para el desarrollo de las entrevistas.

$$
n=\frac{N}{E^{2 *}(N-1)+1}
$$

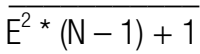

Tabla 1 Delimitación del universo y muestra de empresas con personería jurídica del cantón Antonio Ante.

\begin{tabular}{ccc}
\hline Figura Legal & $\mathrm{N}$ ( Población) & $\mathrm{n}$ ( muestra) \\
\hline Jurídico & 45 & 22 \\
\hline TOTAL & 45 & 22 \\
\hline
\end{tabular}

Es necesario recalcar que el número total de empresas entre personería natural y jurídica del cantón Antonio Ante suma 521 sobre estos datos se estableció el tipo de actividades indicados en la figura 2 y 3 . Además por la naturaleza de la investigación se toma como referencia el subgrupo jurídico con una muestra de 22 empresas, no se ha tomado a la personería natural por constituirse como artesanos calificados y no mostrar el impacto real de factores internos 0 externos que posee la industria.

\section{Instrumentos}

Cuestionario de entrevistas, la estructura del cuestionario para las entrevistas tiene su base en el Plan Nacional del Buen Vivir con preguntas abiertas, donde el entrevistado expresa su opinión propia, permitiendo la obtención de datos no observables que transmitan información acorde al problema planteado en el estudio, dicho instrumento se dirige a; Empresarios del sector textil del cantón Antonio Ante; Sistema financiero del cantón Antonio Ante.

\section{Procedimiento}

La primera técnica de investigación realizada es la observación a través del instrumento de ficha de observación, realizada en la Expo Moda del 
cantón Antonio Ante del 1 al 5 marzo del año 2019 ubicado en el parque Antonio José de Sucre de Atuntaqui calles General Enríquez, Río Amazonas y García Moreno, se toma las variables de evaluación para proceder al análisis de campo, observando variables como; el origen del producto, calidad, precio, segmentación y competencia este proceso llevo a cabo la recopilación de datos que permiten involucrarse de manera directa con el comportamiento del sector textil.

\section{RESULTADOS Y DISCUSIÓN}

En el periodo 2009 al 2017, se evidenció de manera concisa el cambio de la matriz productiva y el modelo de sustitución de importaciones, el periodo 2009-2013, da inicio a la creación del primer Plan Nacional del Buen Vivir, donde se plantea la transformación productiva como estrategia basándose en sectores estratégicos y del 2014-2017, a través del segundo Plan Nacional de Buen Vivir se señala de manera clara, las políticas y lineamientos a realizar para el cambio de la matriz productiva. Se hace hincapié en estos aspectos que serán la base de análisis de los resultados y posterior discusión. (SENPLADES, 2009)

Se analiza la estrategia Transformación del patrón de especialización de la economía, a través de la sustitución selectiva de importaciones para el Buen Vivir, que corresponde al PNBV 2009-2013, aquí se estableció que en este período, según datos proporcionados por el departamento de rentas internas del GAD Municipal de Antonio Ante y reflejados en los gráficos siguientes a la investigación, se presentó un mayor involucramiento a nivel de industrias en áreas como: el agropecuario, financiero, construcción, servicios profesionales y educativo, hotelero, asociaciones, transporte, textil, telecomunicaciones y otros sectores con participaciones del $1 \%$ al $63 \%$, se evidenció que el $23 \%$ correspondió al sector textil. Y en relación a las industrias textiles que formaron parte en este período se determinó un crecimiento del 7\% en comparación a los años siguientes, las actividades más representativas fueron la fabricación de prendas de vestir. La entrevista realizada al sector financiero del cantón Atuntaqui indica que existió una mayor demanda de créditos productivos que fueron invertidos en la adquisición de materias primas, así como en la adecuación y mejora de la infraestructura empresarial.

La estrategia aumento de la productividad real y diversificación de las exportaciones, exportadores y destinos mundiales, se ha dado con la incorporación del valor agregado en el sector textil, que ha permitido el mejoramiento de calidad y aplicación de estrategias encaminadas al crecimiento, desarrollo e innovación de las fábricas textiles, esto demuestra que en el periodo 2009 al 2013 representa el 23\% de la actividad económica total del cantón, en el siguiente periodo se evidencia un decrecimiento en la actividad textil siendo del 16\% con una variación del $7 \%$ debido a factores externos como: la caída del precio de petróleo, competencia desleal entre otros.

La política "Incrementar la productividad por medio de la expansión productiva" y su lineamiento "Formación de enclaves de producción y la construcción de complejos industriales", se analiza desde la visión del Gobierno Autónomo Descentralizado Municipal de Antonio Ante, a través de la entrevista realizada al encargado del Departamento de Gestión de Desarrollo, considera que la fortaleza para influir en el sector textil es apoyo logístico y promoción en ferias textiles que se realiza en la ciudad de Atuntaqui, e indica que la principal amenaza que presenta el sector es la falta de organización entre los miembros de gremios textiles.

Los datos otorgados por el Departamento de Catastro muestran en la figura número 2, el desglose de los diferentes sectores económicos del cantón Antonio Ante, indica que la principal fuente de ingresos del cantón Antonio Ante es el sector textil donde abarca desde la comercialización y fabricación de: prendas de vestir, fibras y telas en el contexto del desarrollo e industrial participa con el $20 \%$. En cuanto a la estructura productiva, en el periodo 2009 al 2017 refleja datos mayores a los demás sectores a pesar de su crecimiento y decrecimiento entre el 2012 al 2017, evidenciando que los años con crecimiento notable son el 2011 y 2014 con 186 y 51 empresas o industrias respectivamente, de igual manera la ubicación de la actividad textil en el periodo 2009 al 2013 del cantón como se observa en la figura número 3 , el desenvolvimiento textil se encuentra en las diferentes parroquias con un $82 \%$ en la ciudad de Atuntaqui, Andrade Marín el 7\%, San Roque 4\%, Natabuela 3\% y Chaltura $4 \%$ a través de las entrevista a los empresarios se determina que la decisión de establecerse en el cantón remota a la tradición 0 emprendimiento y a la potencialidad que presenta la industria textil en el entorno económico brindando empleo a la población.

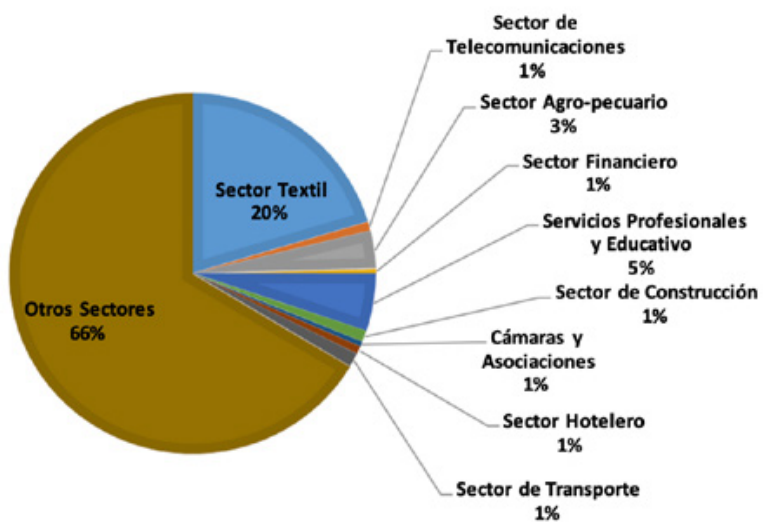

Figura 2. Actividad empresarial por sector del cantón Antonio Ante 2009-2017

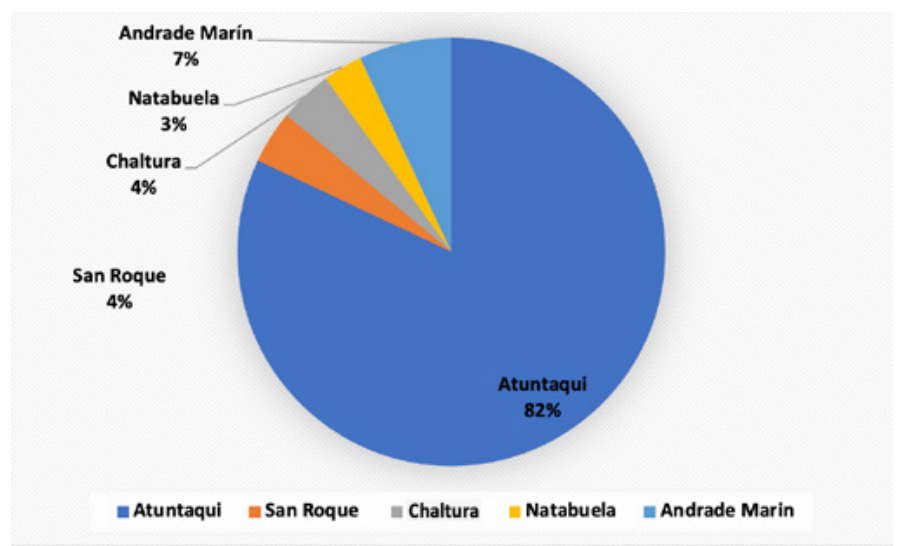

Figura 3. Ubicación de actividad textil en el cantón Antonio Ante 2017

Antes de la crisis de 1929 América Latina crecía al exportar materias primas, sin embargo la demanda mundial de estos bienes disminuyó abruptamente, poniendo fin a la exportación exclusiva de productos primarios, lo que generó un encarecimiento de las importaciones, por lo que el postulado de Prebisch sobre la caída de los términos de intercambio, influye para que países periféricos ya no se subordinen a grandes economía y apostar más bien por el fomento de la industrialización, con el accionar directo del Estado, en el caso ecuatoriano con la sustitución de importaciones y cambio de la matriz productiva.

A través de la investigación realizada se ha logrado establecer la incidencia, con la identificación de aspectos, que permitan reconocer las estrategias tomadas por el gobierno para la mejora de crecimiento y rentabilidad en el sector productivo, principalmente textil, tomando referencia al cantón Antonio Ante. Es así que el cambio de matriz pro- 
ductiva para su cumplimiento fija las bases de transformación en los sectores estratégicos, que poseen alto valor agregado identificando la capacidad productiva para el crecimiento de exportaciones, conllevando a lograr una adecuada sustitución de importaciones, con la meta de mejorar la calidad de vida, la estructura productiva e independencia del consumo externo.

Ecuador para lograr el adecuado cambio de la estructura productiva enfrentó factores como el valor agregado a productos exportables, beneficios directos a sectores tradicionales, desigualdad de acceso a la inversión extranjera y crediticia nacional, entre otros. Estos instrumentos pretenden generar la correcta aplicación de estrategias para transformar la matriz productiva del Ecuador, creando competitividad externa especialmente en la región, garantizando el crecimiento económico e industrial a través del cambio de una estructura tradicional a moderna.

Las políticas trasversales juegan un papel importante en la disminución de brechas socioeconómicas, con ayuda del trabajo conjunto entre la industria pública y privada. Brinda seguridad en el cumplimiento de objetivos propuestos por el Estado creando modelos eficientes de desarrollo organizativo de las nuevas industrias. Las presentes políticas van desde la innovación, comercio, calidad, capacitación, inversión y emprendimiento, cada una de estas cumple un objetivo propio para el sector manufacturero o intermedio, permite el mejoramiento constante de la cadena de producción, a la vez el tema sectorial es importante con el involucramiento de entidades del Estado, abarcando todo nivel, desde el ambiental, industrial tributario, social, económico, comercial y promocional, esto permite solucionar problemas que afecta al entorno empresarial como la tramitología de permisos, licencias, solicitudes, patentes, procesos de exportación e importación entre otros reduciendo tiempo y costo.

Los instrumentos y políticas establecidas buscan la mejora constante de los sectores económicos principalmente de los no tradicionales, donde se encuentra el manufacturero como subgrupo de la fabricación de prendas de vestir, productos textiles, elaboración de cuero y derivados que conlleva a determinar que las propuestas no cumplen con el objetivo dado, debido la existencia de falta de información por parte de instituciones hacia empresarios, conlleva al desequilibrio de la participación productiva en el caso del sector textil del cantón Antonio Ante, en el que se evidencia la desconformidad debido a que las empresas constituidas como figuras jurídicas, representan el 9\% en comparación al 91\% de la sociedad natural en el periodo 2009-2017, debido a que la sociedad jurídica demuestra mayor crecimiento en la infraestructura, maquinaria, capital fijo e involucramiento en el mercado local y nacional, inclusive internacional, es así que no cumple con el requerimiento indicado en la Ley de Defe (Silvia, 2018)nsa del Artesano artículo 2, al manifestar que el artesano es la persona que "desarrolle su actividad y trabajo personalmente y hubiere invertido en su taller, en implementos de trabajo, maquinarias y materias primas, una cantidad no superior al veinticinco por ciento $(25 \%)$ del capital fijado para la pequeña industria" (Junta Nacional de Defensa del Artesano, 2008, p. 1), al no ser considerado como tal no acceden a los beneficios otorgados por el gobierno como; exoneración del impuesto a la renta, importaciones de materiales e insumos para la proceso productivo con beneficios arancelarios, prestación de créditos financieros de manera accesible con tasas de interés y plazos favorables.

Ecuador posee una estructura arancelaria evidente en la industria textil, donde abarca desde el capítulo 50 al 63, consolidando el manejo de las paridades en el contexto comercial a través del uso de materias primas y procesadas, es así que en este periodo el gobierno establece medidas no arancelarias que permiten la estimulación de la producción y comercio con el propósito de proteger a la industria nacional, por lo que el 22 de enero del 2009 se establece salvaguardias a 1250 partidas arancelarias de manera general, tomando a 261 subpartidas arancelarias que favorecen al sector textil en esencial a las prendas de vestir, dicha estrategia ayudó de manera directa a la industria productora, pero originó conflictos a los comercializadores e importadores de prendas de vestir. (AITE, 2016)

En el caso de estudio del cantón Antonio Ante, la medida tomada por el gobierno favoreció de manera directa a la industria de confección, logrando establecer estrategias de mercado que brinden equilibrio al consumo de productos nacionales. Pero el sector debe enfrentar sobretasas arancelarias en insumos adquiridos en el mercado internacional, debido a que la producción de materia prima nacional no cubre la demanda requerida para el proceso de fabricación, por lo cual el sector realiza importaciones. Dicha sobre- tasa aplica a 825 sub partidas textiles con el pago porcentual del $5 \%$ y $45 \%$ adicional al valor del bien adquirido en el mercado externo. En los siguientes años el crecimiento del sector se ve afectado por factores ocasionados, por la eliminación de las salvaguardias ocasionando el incremento del contrabando, incremento de importaciones de prendas de vestir, materia prima e insumos a precios inferiores de los costos de producción y competencia desleal. (Cumba, 2016)

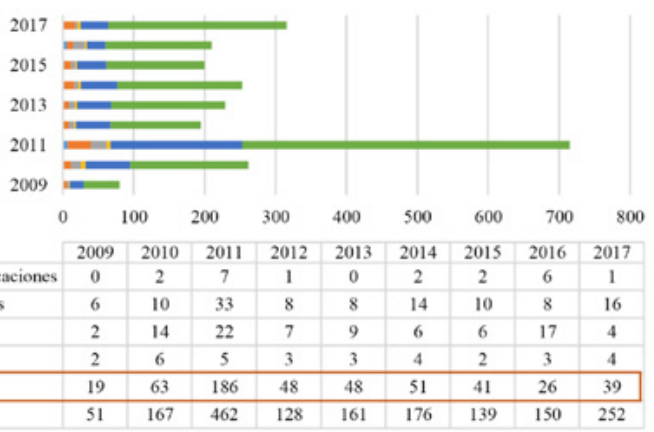

Figura 4. Variación sectores económicos del cantón Antonio Ante 2009 - 2017

La propuesta del gobierno en promover el cambio de la matriz productiva establecida en el objetivo diez del PNVB del 2013 al 2017, dictamina una mayor concentración de los sectores estratégicos a través de políticas y lineamientos, es así que el sector textil participa en las acciones que son; sustitución de importaciones, beneficios tributarios, diversificación de exportaciones, capacitación al recurso humano, mejoramiento en la infraestructura y equipos de productividad, por último acceder a las compras públicas textiles realizados por el gobierno. La primer acción de sustitución de importaciones es clave para lograr el desarrollo del sector, pero como se ha mencionado sobre la falta de demanda requerida, conlleva a que la industria no logre dicha propuesta, debido a que el valor en miles de dólares FOB de las importaciones es de \$ 4.915.968 en relación a las exportaciones de $\$ 1.375 .738$ según datos del Banco Central del Ecuador (BCE) en el periodo 2009 al 2016 la diferencia de los valores, permiten determinar que el sector realiza mayor adquisición de bienes extranjeros ya sea materia prima, insumos o productos confeccionados.

\section{CONCLUSIONES}

Los postulados de Prebisch adoptados por la CEPAL constituyen la base de origen que establece las fases para el desarrollo y crecimiento de los países periféricos a través del cambio productivo y la adecuada sustitución de importaciones, en donde busca generar independencia de pro- 
ductos o servicios elaborados por países desarrollados, promoviendo la necesidad de edificar políticas que beneficien a los sectores no tradicionales dejando de ser conocidos como distribuidores de materia prima.

Los organismos del gobierno, encargados de buscar el crecimiento económico del país en función de los instrumentos creados, para el cambio de la matriz productiva, promueven una sustitución de importaciones, con la vinculación entre Estado e industria privada, a través de la contratación pública, participación de ferias que promueven la oferta y demanda del producto nacional entre otros. Dichos mecanismos no son considerados por propietarios de la industria textil del cantón, debido a que no va en función de la necesidad requerida o la falta de diálogo entre actores.

Se determinan la existencia de factores negativos que afectan en gran medida al sector textil, en esencial la falta de control por autoridades a la competencia desleal que atraviesa el mercado por productos que ingresan de manera ilícita.

La industria textil del cantón Antonio Ante se ha visto afectado durante el periodo de estudio, con una disminución porcentual de participación del 23\% (2009 -2013) al 16\% (2014-2017), como consecuencia de las reformas realizadas en el contexto laboral, comercial y productivo, sobre todo la liberalización del mercado.

El cambio de la matriz productiva y el modelo de sustitución de importaciones en el ámbito textil, favorece de manera desigualitaria en relación a otros sectores, a causa de la falta de acuerdos comerciales, inversión extranjera y políticas que beneficie de manera apropiada y directa a dicho sector, debido a que el gobierno desplaza a los sectores priorizados basando su balanza comercial en los productos que no poseen valor agregado.

Por último, la presente investigación tuvo como uno de sus fines analizar la implementación de políticas encaminadas al cambio de la matriz productiva, en función al sector textil, donde se determina que actualmente el gobierno no promueve el crecimiento de los sectores priorizados, manteniendo estrategias encaminadas a la economía tradicional desprotegiendo a la industria manufacturera.

\section{REFERENCIAS BIBLIOGRÁFICAS}

AITE. (2016). Asociación de Industriales Textiles del Ecuador. Recuperado el 12 de marzo de 2019, de https://www.aite.com.ec/boletines/2016/industria-textil.pdf

Artesano, J. N. (14 de mayo de 2008). Junta Nacional de Defensa del Artesano. Recuperado el 19 de febrero de 2019, de http://extwprlegs1.fao.org/docs/pdf/ecu168907.pdf

Cumba, E. d. (2016). El crecimiento de la industria textil en Atuntaqui en el periodo 2005- 2010: el papel del Gobierno de Antonio Ante. Recuperado el 15 de febero de 2019, de http://repositorio.puce. edu.ec/bitstream/handle/22000/12357/Disertaci\%C3\%B3n_ Estefan\%C3\%ADa_Ulloa_PDF.pdf

Garzón , N., Kulfas, M., Palacios, J., \& Dirchelmo, T. (1 de 05 de 2016). INEC. Recuperado el 27 de enero de 2019, de https://www. ecuadorencifras.gob.ec/documentos/web-inec/Bibliotecas/Libros/SECTOR\%20MANUFACTURERO.pdf

Prebisch, R. (2012). CEPAL. Recuperado el 17 de marzo de 2019, de https://www.cepal.org/es/publicaciones/40010-desarrollo-economico-la-america-latina-algunos-sus-principales-problemas

SENPLADES. (2009). SENPLADES. Recuperado el 18 de abril de 2019, de http://www.ecuadorencifras.gob.ec/wp-content/
descargas/\%20InformacionLegal/Normas-de-Regulacion/ Plan-Nacional-para-el-BuenVivir/Plan+Nacional+del+Buen+Vivir+2009-2013.pdf

Silvia, T. P. (2018). Análisis de la incidencia del sector textil en la economía nacional 2013-2017. Recuperado el 28 de marzo de 2019, de http://repositorio.ug.edu.ec/bitstream/redug/34513/1/ SILVA\%20TAYO.pdf 